\title{
On Confinement of Fermions in Strongly Coupled Lattice Gauge Theory
}

\author{
K. Gawędzki *
}

Max-Planck-Institut für Physik und Astrophysik, D-8000 München 40, Federal Republic of Germany

\begin{abstract}
A lattice theory of Fermi fields of mass $m$ coupled to gauge fields in the region where $m$ and the gauge field coupling constant $g$ are large is studied. It is shown that the energy of some states composed of a fermion and a distant antifermion with a string in between grows at least linearly with the distance if $1<g^{6}<m<g^{\varepsilon \log g}$.
\end{abstract}

\section{Introduction}

The lattice gauge field theory was formulated by Wilson [15] with the hope that the mechanisms behind the long distance behavior of the continuum fields could be understood in the simplified lattice models, see also $[1,7]$. The primary aim was to understand the quark confinement. Working with the QED Wilson formulated a criterion for charge confinement which involved only the electromagnetic field (no Fermi fields):

if $\left\langle\exp \left(\right.\right.$ ie $\left.\left.\int_{\partial s} A_{\mu} d x^{\mu}\right)\right\rangle \sim \exp (-C(s))$ and $C(s)$ is proportional

to the area of the two-dimensional cube $s$ then charge should be confined; if $C(s)$ is proportional to the circumference of $s$ then no confinement occurs.

The criterion was based on the analysis of the expansion of Euclidean propagators of full lattice QED into powers of, say, inverse fermion mass, interpreted as a sum over fermion-antifermion trajectories. Each path $\sigma$ contributed a lattice version of $\left\langle\exp \left(i e \int_{\sigma} A_{\mu} d x^{\mu}\right)\right\rangle$. It was argued that in the case of the "area law", paths with fermion and antifermion well separated hardly entered. Wilson suggested that in the lattice QED the area law should hold for large coupling constant $g$. This was confirmed by the rigorous result of OsterwalderSeiler [10] obtained for a wide class of lattice gauge theories. In the meantime

* On leave from Department of Mathematical Methods of Physics, Warsaw University, Hoża 74 , PL 00-682 Warsaw, Poland 
other possible mechanisms leading to the area law have been proposed, believed to work for weak or intermediate coupling (instantons, merons) $[2,3,6,12,13]$.

In the present paper we consider a gauge field interacting with a color multiplet of Dirac Fermi fields (in Euclidean region). Our result is a rigorous step in proving occurence of confinement for large Dirac fields mass $m$ and large coupling constant $g$, starting from a different criterion, more appealing to the physical situation. It may be also viewed however as a rigorous step on the way to substantiate the Wilson's criterion.

The criterion we use is based on the following rough idea : confinement occurs if the physical (i.e. gauge invariant!) states with gauge charges concentrated in well separated regions have big energy, growing with the separation. This idea of confinement, where the use of gauge invariant states is a crutial point, is very close to the one used in [7]. Our test-states $X_{r}$ consist of a fermion and an antifermion connected by a gauge field string (necessary to have gauge invariance). We are able to prove that once the spins of the fermion and the antifermion are correlated in a certain way the energy of the state grows at least linearly in $r$ for some $m \gg g \gg 1$. The correlation between spins of the pair seems to result only from our inability to dismiss this assumption.

The basic technical tool we use is the cluster expansion - a generalization of the one worked out in $[9,10]$ for pure lattice Yang-Mills theory (without fermions). Section 2 is devoted to the formulation and to the proof of convergence of the cluster expansion. As usually, once the convergence is proven, existence of the exponentially clustering infinite volume theory follows and the standard construction [11] gives the physical Hilbert space and the transfer matrix $e^{-2 I I}$, since the Osterwalder-Schrader positivity holds, as proven in [10].

The confinement bound is deduced from the estimate

$$
\frac{1}{\left\|X_{r}\right\|^{2}}\left(X_{r} \mid e^{-2 H} X_{r}\right) \leqq e^{-0(1) r} .
$$

To obtain (1) we bound $\left(X_{r} \mid e^{-2 H} X_{r}\right)$ from above by $e^{-C_{1} r}$ directly from the cluster expansion. The missing lower bound on $\left\|X_{r}\right\|{ }^{2},\left\|X_{r}\right\|^{2} \geqq e^{-C_{2} r}$, with $C_{2}<C_{1}$, is more difficult. One can bound $\left\|X_{r}\right\|^{2}$ from below computing it in the lowest order of the strong coupling perturbation calculus and estimating the correction by the cluster expansion, which is well suited for that. However this does not lead to the searched bound directly. Nevertheless this bound can be obtained if we use additionally a convexity of $\log \left\|X_{r}\right\|$ (Proposition 2) which can be proven by a sort of Nelson symmetry argument. This is where our nasty assumption on correlation of spins of the fermion and the antifermion enters. The estimates leading to the lower linear bound on the energy of $X_{r}$ compose Section 3 of the paper.

\section{Cluster Expansion}

The model we study is essentially the same as the one considered by OsterwalderSeiler [10, Sections II.2 and II.3]. We shall briefly recall and supplement their notation, introducing some minor changes. 
Let $\Lambda$ be a finite subset in the hypercubic $d$-dimensional lattice $\mathbb{L}=\left(\frac{1}{2}, \ldots, \frac{1}{2}\right)$ $+\mathbb{Z}^{4} \subset \mathbb{R}^{4}$. By $B_{A}$ we shall denote the set of oriented bonds $b$ [pairs $\left(x_{b}, y_{b}\right)$ of nearest neighbor sites] in $A$, by $B_{A}^{+}$the set of bonds in $\Lambda$ with positive orientation (with respect to the coordinate axes) and by $P_{A}$ the set of all oriented plaquettes $p$ (lattice squares) in $\Lambda$. The basic Euclidean field variables are:

$$
\psi_{\alpha A}^{i}(x) \quad i=1,2, \quad \alpha=0,1,2,3, \quad A=1, \ldots, N, \quad x \in \mathbb{L},
$$

(they generate a Grassmann algebra) and

$$
g_{b} \equiv g_{b-1}^{-1} \in G,
$$

where $b, b^{-1}$ are bonds, $b^{-1}=\left(y_{b}, x_{b}\right)$, and $G$ is the gauge group taken to be $U(1)$ or $\mathrm{SU}(n)$.

The Euclidean fermionic action in finite volume is taken to be (compare [10])

$$
\begin{aligned}
A_{A}^{F}:= & \sum_{x \in A} \sum_{\alpha=0}^{3} \sum_{A=1}^{N} m \psi_{\alpha A}^{2}(x) \psi_{\alpha A}^{1}(x)-\frac{1}{2} \sum_{b \in B_{A}} \sum_{\alpha, \beta=0}^{3} \sum_{A, B=1}^{N} \psi_{\alpha A}^{2}\left(x_{b}\right) \\
& \cdot\left(\gamma_{b}^{E}\right)_{\alpha \beta} U\left(g_{b}\right)_{A B} \psi_{\beta B}^{1}\left(y_{b}\right) .
\end{aligned}
$$

Here $U$ is a fixed $N$-dimensional unitary representation of $G, \gamma_{b}^{E}=\gamma_{\alpha}^{E}$ if $b \in B_{A}^{+}$and $\gamma_{b}^{E}=-\gamma_{\alpha}^{E}$ otherwise, where $\alpha$ corresponds to the coordinate axis parallel to $b$, and the Euclidean Dirac matrices are chosen as in [10].

The Euclidean gauge field action is given by

$$
A_{\Lambda}^{Y M}:=-\frac{1}{2 g^{2}} \sum_{p \in P_{A}} \chi\left(g_{p}\right),
$$

where $g_{p}$ is the product of four bond variables along the boundary of $p$ (defined up to conjugacy class) and $\chi$ is a character of $G$ (trace of a $D$-dimensional unitary representation).

Let $\mathscr{A}_{A}=\oplus \mathscr{A}_{A}^{r}$ denote the Grassmann algebra generated by $\left(\psi_{\alpha A}^{i}(x)\right)_{x \in A}\left(\mathscr{A}_{A}^{r}\right.$ being its subspace of order $r$ ). Similarly as in [10] we let \langle\rangle$_{A}^{F}$ denote the linear functional on $\mathscr{A}_{A}$ defined by $\left\langle\mathscr{A}_{A}^{r}\right\rangle_{A}^{F}=0$ if $r$ is not maximal,

$$
\left\langle\prod_{\substack{\alpha, A \\ x \in A}} \psi_{\alpha A}^{2}(x) \psi_{\alpha A}^{1}(x)\right\rangle_{A}^{F}=1 .
$$

Consider the space $\mathfrak{U}_{A}$ of the continuous mappings from $\underset{b \in B_{\Lambda}^{+}}{X} G$ into $\mathscr{A}_{\Lambda}$. For $\mathscr{F} \in \mathfrak{U}_{A}$ define

$$
\langle\mathscr{F}\rangle_{A}:=\frac{\int d g_{A}\left\langle\mathscr{F} e^{-A^{F}}\right\rangle_{A}^{F} e^{-A^{Y M}}}{\int d g_{A}\left\langle e^{-A^{F}}\right\rangle_{A}^{F} e^{-A^{Y M}}},
$$

where $d g_{\Lambda}=\bigotimes_{b \in B_{A}^{+}} d g, d g$ being the normalized Haar measure on $G$. 
Now a local gauge transformation $\gamma \in \underset{x \in \mathbb{L}}{X} G$ acts on $\underset{b \in B_{A}^{+}}{X} G$ by $\left(g_{b}\right) \mapsto\left(g_{b}^{\gamma}\right)$

$g_{b}^{\gamma}=\gamma_{x_{b}} g_{b} \gamma_{v_{b}}^{-1}$

and also defines an authomorphism of $\mathscr{A}_{A}$ :

$$
\begin{aligned}
& \gamma \psi_{\alpha A}^{1}(x)=\sum_{B} U\left(\gamma_{x}^{-1}\right)_{A B} \psi_{B}^{1}(x), \\
& \gamma \psi_{\alpha A}^{2}(x)=\sum_{B} \overline{U\left(\gamma_{x}^{-1}\right)_{A B}} \psi_{B}^{2}(x) .
\end{aligned}
$$

These actions induce an authomorphism on $\mathfrak{P H}_{A} \mathscr{F} \mapsto \mathscr{F} v$

$$
\mathscr{F}^{\gamma}\left(\left(g_{b}\right)\right):=\gamma \mathscr{F}\left(\left(g_{b}^{\gamma^{-1}}\right)\right) \text {. }
$$

The subspace of $\mathfrak{H}_{A}$ of invariant elements will be denoted by $\mathfrak{H}_{A}^{\text {inv }}$. \langle\rangle$_{A}$ is invariant under local gauge transformations:

$$
\left\langle\mathscr{F}^{\gamma}\right\rangle_{A}=\langle\mathscr{F}\rangle_{A} \text {. }
$$

Take

$$
\mathscr{F}=\prod_{i=1}^{l} \psi_{\alpha_{1} A_{i}}^{2}\left(x_{i}\right) \psi_{\beta_{1} B_{1}}^{1}\left(y_{i}\right) \mathscr{G},
$$

where $\mathscr{G}$ is a complex continuous function on $\underset{b \in B_{A}^{+}}{X} G$. Standard computation of Gaussian anticommuting integral gives a sort of Matthews-Salam formula

$$
\begin{aligned}
\langle\mathscr{F}\rangle_{A}= & \frac{1}{Z_{A}} \int d g_{A}\left(\frac{-1}{m}\right)^{l} \operatorname{det}_{i_{1} l_{2}}^{l \times l}\left[\left(1-\frac{1}{m} K_{A}\right)_{\beta_{1_{1}} B_{1_{1}} y_{1_{1}}, \alpha_{i_{2}} A_{l_{2}, \lambda_{l_{2}}}}^{-1}\right] \\
& \cdot \operatorname{det}\left(1-\frac{1}{m} K_{A}\right) \mathscr{G} e^{-A_{A}^{X M}},
\end{aligned}
$$

where

$$
Z_{A}=\int d g_{A} \operatorname{det}\left(1-\frac{1}{m} K_{A}\right) e^{-A_{A}^{Y M}}
$$

and $K_{A}$ is a matrix,

$$
\left(K_{A}\right)_{\alpha A x, \beta B y}=\left\{\begin{array}{l}
\frac{1}{2}\left(\gamma_{(x, y)}^{E}\right)_{\alpha \beta} U\left(g_{(x, y)}\right)_{A B} \\
0 \quad \text { otherwise. }
\end{array}\right.
$$

To be sure that (4) and (9) make sense we have to show still that $Z_{A} \neq 0$. This follows immediately from

Lemma 1. $\operatorname{det}\left(1-\frac{1}{m} K_{\Lambda}\right)>0$.

Proof of Lemma. We have

$$
K_{A}^{*}=-K_{A}=\gamma_{5}^{E} K_{A} \gamma_{5}^{E}
$$


where

$$
\gamma_{5}^{E}=\gamma_{0}^{E} \gamma_{1}^{E} \gamma_{2}^{E} \gamma_{3}^{E}=\left(\gamma_{5}^{E}\right)^{*}=\left(\gamma_{5}^{E}\right)^{-1}
$$

Hence

$$
\overline{\operatorname{det}\left(1-\frac{1}{m} K_{\Lambda}\right)}=\operatorname{det}\left(1-\frac{1}{m} K_{\Lambda}^{*}\right)=\operatorname{det}\left(1-\frac{1}{m} K_{\Lambda}\right)
$$

and the determinant is real. Moreover as $K_{\Lambda}$ is antihermitian, $1-\frac{1}{m} K_{A}$ cannot have eigenvalue zero. Since $\lim _{m \rightarrow \infty} \operatorname{det}\left(1-\frac{1}{m} K_{\Lambda}\right)=1$, $\operatorname{det}\left(1-\frac{1}{m} K_{\Lambda}\right)$ must be always positive.

We shall examine the theory in the region where $m$ and $g$ are large (strong coupling) using a cluster expansion generalizing the one used in $[9,10]$ for lattice Yang-Mills fields only. The generalization is patterned on the cluster expansion used in the continuum Yukawa model $(\mathrm{Yu})_{2}$, see $[4,8]$ but again is much simpler because we work on the lattice. It will resemble however the cluster expansions for continuum models, especially of $[6,4]$, more than the expansion of $[10]$, since we shall be turning off the non-local parts of interaction smoothly.

To this end introduce for each function

$$
s: B_{A} \rightarrow[0,1] \text { and } \tau: P_{A} \rightarrow[0,1]
$$

interpolating objects $K_{A s}$ and $A_{A \tau}^{Y M}$ by

$$
\begin{aligned}
& \left(K_{\Lambda s}\right)_{\alpha A x, \beta B y}:= \begin{cases}\frac{1}{2} s((x, y))\left(\gamma_{(x, y)}^{E}\right)_{\alpha \beta} U\left(g_{(x, y)}\right)_{A B} \text { if }(x, y) \text { is a bond in } \Lambda, \\
0 & \text { otherwise, }\end{cases} \\
& A_{\Lambda \tau}^{Y M}:=\frac{1}{2 g^{2}} \sum_{p \in P_{A}} \tau(p) \chi\left(g_{p}\right) .
\end{aligned}
$$

For $\Gamma \subset B_{\Lambda}, Q \subset P_{\Lambda}$ and $s, \tau$ as above define $s_{\Gamma}, \tau_{Q}$ by

$$
s_{\Gamma}(b):=\left\{\begin{array}{l}
s(b) \text { if } b \in \Gamma, \\
0 \text { otherwise }
\end{array}\right.
$$

and analogically for $\tau_{Q}$. The first step in the expansion is (compare [6]):

$$
\begin{aligned}
\langle\mathscr{F}\rangle_{\Lambda}= & \frac{1}{Z_{A}} \sum_{\substack{\Gamma \subset B_{\Lambda} \\
Q \subset \boldsymbol{P}_{\Lambda}}} \int d s_{\Gamma} d \tau_{Q} \partial_{s_{\Gamma}} \partial_{\tau_{Q}} \int d g_{\Lambda}\left(\frac{-1}{m}\right)^{l} \\
& \cdot \operatorname{det}_{i_{1} i_{2}}^{l \times l}\left[\left(1-\frac{1}{m} K_{\Lambda s_{\Gamma}}\right)_{\boldsymbol{y}_{i_{1}}, \boldsymbol{x}_{i_{2}}}^{-1}\right] \operatorname{det}\left(1-\frac{1}{m} K_{\Lambda s_{\Gamma}}\right) \mathscr{G} e^{-A_{\Lambda}^{\tau^{\prime} \boldsymbol{Q}}} .
\end{aligned}
$$


Here $\int d s_{\Gamma} d \tau_{Q}=\prod_{b \in \Gamma} \int d s(b) \prod_{p \in Q} \int d \tau(p), \partial_{s_{\Gamma}} \partial_{\tau_{Q}}=\prod_{b \in \Gamma} \frac{\partial}{\partial s(b)} \prod_{p \in Q} \frac{\partial}{\partial \tau(p)}, \boldsymbol{y}_{i_{1}}$ stands for $\beta_{i_{1}} B_{i_{1}} y_{i_{1}}$ and $\boldsymbol{x}_{i_{2}}$ for $\alpha_{i_{2}} A_{i_{2}} x_{i_{2}}$. The terms with $\Gamma=\emptyset$ or $Q=\emptyset$ also enter (there is no integration and differentiations involving $s_{\Gamma}$ or $\tau_{Q}$ then).

Now define

$$
\operatorname{supp} \mathscr{F}:=\bigcup_{i=1}^{l}\left\{x_{i}, y_{i}\right\} \cup \operatorname{supp} \mathscr{G}
$$

where $\operatorname{supp} \mathscr{G}$ is the union of lattice sites - ends of bonds $b$ such that $\mathscr{G}$ depends non-trivially on $g_{b}$. To $(\Gamma, Q)$ assign the subset $\overline{\Gamma \cup Q}$ in $R^{d}$ composed of closed unit intervals corresponding to the bonds in $\Gamma$ and to the boundary bonds of the plaquettes in $Q$. Suppose that $\Gamma=\Gamma_{0} \cup \Gamma_{1}, Q=Q_{0} \cup Q_{1}, \Gamma_{0} \cap \Gamma_{1}=\emptyset, Q_{0} \cap Q_{1}=\emptyset$ and that $\overline{\Gamma_{1} \cup Q_{1}}$ is disjoint from $\overline{\Gamma_{0} \cup Q_{0}} \cup \operatorname{supp} \mathscr{\mathscr { F }}$. Then the right hand side of (14) "decouples":

$$
\begin{aligned}
& \int d s_{\Gamma} d \tau_{Q} \partial_{S_{\Gamma}} \partial_{\tau_{Q}} R d g_{\Lambda}\left(\frac{-1}{m}\right)^{l} \operatorname{det}_{i_{1} i_{2}}^{l \times l}\left[\left(1-\frac{1}{m} K_{\Lambda s_{\Gamma}}\right)_{\boldsymbol{y}_{i_{1}}, \boldsymbol{x}_{i_{2}}}^{-1}\right] \\
& \cdot \operatorname{det}\left(1-\frac{1}{m} K_{\Lambda s_{\Gamma}}\right) \mathscr{G} e^{-A_{A \tau_{Q}}^{Y M}} \\
& =\left(\int d s_{\Gamma_{0}} d \tau_{Q_{0}} \partial_{s_{\Gamma_{0}}} \partial_{\tau_{Q_{0}}} \int d g_{\Lambda}\left(\frac{-1}{m}\right)^{l} \operatorname{det}_{i_{1} i_{2}}^{l \times l}\left[\left(1-\frac{1}{m} K_{\Lambda s_{\Gamma_{0}}}\right)_{\boldsymbol{y}_{i_{1}} \boldsymbol{x}_{i_{2}}}^{-1}\right]\right. \\
& \left.\cdot \operatorname{det}\left(1-\frac{1}{m} K_{\Lambda s_{\Gamma_{0}}}\right) \mathscr{G}^{-A \tau_{\Lambda \tau_{Q_{0}}}^{Y M}}\right) \cdot\left(\int d s_{\Gamma_{1}} d \tau_{Q_{1}} \partial_{S_{\Gamma_{1}}} \partial_{\tau_{Q_{1}}} \int d g_{\Lambda}\right. \\
& \left.\cdot \operatorname{det}\left(1-\frac{1}{m} K_{\Lambda s_{\Gamma_{1}}}\right) e^{-A \tau_{\tau_{Q_{1}}}^{Y M}}\right) .
\end{aligned}
$$

Suppose that from all possible divisions of $\Gamma$ and $Q$ described above we always choose that leading to minimal $\overline{\Gamma_{0} \cup Q_{0}}$. Using (15) we may perform in (14) a partial resummation fixing $\left(\Gamma_{0}, Q_{0}\right)$ and summing over all $\left(\Gamma_{1}, Q_{1}\right)$ and only then summing over all $\left(\Gamma_{0}, Q_{0}\right), \Gamma_{0} \subset B_{\Lambda}, Q_{0} \subset P_{A}$, such that $\overline{\Gamma_{0} \cup Q_{0}}$ has no connected component disjoint from supp $\mathscr{F}$. This leads to

$$
\begin{aligned}
\langle\mathscr{F}\rangle_{A}= & \sum_{\left(\Gamma_{0}, Q_{0}\right)} \int d s_{\Gamma_{0}} d \tau_{Q_{0}} \partial_{s_{\Gamma_{0}}} \partial_{\tau_{Q_{0}}} \int d g_{\Lambda}\left(\frac{-1}{m}\right)^{l} \\
& \cdot \operatorname{det}_{i_{1} i_{2}}^{l \times l}\left[\left(1-\frac{1}{m} K_{\Lambda s_{\Gamma_{0}}}\right)_{\boldsymbol{y}_{i_{1}}, \boldsymbol{x}_{i_{2}}}\right] \\
& \cdot \operatorname{det}\left(1-\frac{1}{m} K_{\Lambda s_{\Gamma_{0}}}\right) \mathscr{G}^{-A_{\Lambda \tau_{Q_{0}}}} \frac{Z_{\left.\Lambda \backslash \overline{\Gamma_{0} \cup Q_{0}} \cup \operatorname{supp} \mathscr{F}\right)}}{Z_{\Lambda}} \\
& \equiv \sum_{\left(\Gamma_{0}, Q_{0}\right)} R\left(\mathscr{F}, \Gamma_{0}, Q_{0}\right) \frac{Z_{\Lambda \backslash\left(\overline{\Gamma_{0} \cup Q_{0}} \cup \operatorname{supp} \mathscr{F}\right)}}{Z_{\Lambda}} .
\end{aligned}
$$


Let $K:=\left|\Gamma_{0}\right|, L:=\left|Q_{0}\right|, \Gamma_{0}=\left\{b_{l+1}, \ldots, b_{l+K}\right\}, b_{k}=\left(x_{k}, y_{k}\right), k=l+1, \ldots, l+K$. An easy calculation, which we leave to the reader, gives

$$
\begin{aligned}
R\left(\mathscr{F}, \Gamma_{0}, Q_{0}\right)= & \int d s_{\Gamma_{0}} d \tau_{Q_{0}} \frac{1}{2^{K+L}}\left(\frac{-1}{m}\right)^{l+K}\left(\frac{1}{g^{2}}\right)^{L} \int d g_{\Lambda} \\
& \cdot \sum_{\substack{\alpha_{k}, \beta_{k}, A_{k}, B_{k} \\
k=l+1, \ldots, l+K}}\left(\prod_{k}\left(\gamma_{b_{k}}^{E}\right)_{\alpha_{k} \beta_{k}} U\left(g_{b_{k}}\right)_{A_{k} B_{k}}\right) \operatorname{det}_{i_{1} i_{2}}^{(l+K) \times(l+K)} \\
& \cdot\left[\left(1-\frac{1}{m} K_{A s_{\Gamma_{0}}}\right)_{\boldsymbol{y}_{t_{1}}, \boldsymbol{x}_{\boldsymbol{t}_{2}}}^{-1}\right] \operatorname{det}\left(1-\frac{1}{m} K_{\Lambda s_{\Gamma_{0}}}\right) \mathscr{G} \prod_{p \in Q_{0}} \chi\left(g_{p}\right) e^{-A_{A \tau_{Q_{0}}}^{X M}} .
\end{aligned}
$$

Throughout the paper we shall denote by $0(1)$ various constants, which can depend only on $N$ and $D$.

Lemma 2. If $m, g>1$ then

$$
\left|R\left(\mathscr{F}, \Gamma_{0}, Q_{0}\right)\right| \leqq\left(\frac{e}{m}\right)^{l}\|\mathscr{G}\|_{\infty} e^{0(1)(K+L)}\left(\frac{1}{m}\right)^{K}\left(\frac{1}{g^{2}}\right)^{L} .
$$

Proof of Lemma.

$$
\left|\operatorname{det}_{i_{1} i_{2}}^{(l+K) \times(l+K)}\left[\left(1-\frac{1}{m} K_{\Lambda s_{\Gamma_{0}}}\right)_{\boldsymbol{y}_{l_{1}}, \boldsymbol{x}_{i_{2}}}^{-1}\right] \operatorname{det}\left(1-\frac{1}{m} K_{\Lambda s_{\Gamma_{0}}}\right)\right| \leqq e^{l+K+\frac{1}{m}\left\|K_{\Lambda s_{T_{0}}}\right\|_{1}}
$$

by the Cauchy integral formula, since the left hand side is equal

$$
\left|\frac{\partial^{l+K}}{\partial \mu_{1} \ldots \partial \mu_{l+K}}\right|_{0} \operatorname{det}\left(1-\frac{1}{m} K_{\Lambda s_{I_{0}}}+\mu_{1} B_{1}+\ldots+\mu_{l+K} B_{l+K}\right),
$$

where

$$
\left(B_{i}\right)_{\boldsymbol{x}, \boldsymbol{y}}:=\delta_{\boldsymbol{x}, \boldsymbol{x}_{i}} \delta_{\boldsymbol{y}_{i}, \boldsymbol{y}},
$$

and

$$
|\operatorname{det}(1+C)| \leqq e^{\|C\|_{1}} .
$$

But

$$
\left\|K_{\Lambda s_{\Gamma_{0}}}\right\|_{1} \leqq \frac{4 N}{2} K .
$$

Hence

$$
\begin{aligned}
& \mid \sum_{\substack{\alpha_{k}, \beta_{k}, A_{k}, B_{k} \\
k=l+1, \ldots, l+K}}\left(\prod_{k}\left(\gamma_{b_{k}}^{E}\right)_{\alpha_{k} \beta_{k}} U\left(g_{b_{k}}\right)_{A_{k} B_{k}}\right) \\
& \cdot \operatorname{det}_{i_{1} i_{2}}^{(l+K) \times(l+K)}\left[\left(1-\frac{1}{m} K_{A s_{S_{0}}}\right)_{\boldsymbol{y}_{i_{1}}, \boldsymbol{x}_{i_{2}}}^{-1}\right] \operatorname{det}\left(1-\frac{1}{m} K_{A s_{\Gamma_{0}}}\right) \mid \\
& \leqq \prod_{k}\left(\sum_{\alpha_{k}, \beta_{k}}\left|\left(\gamma_{b_{k}}^{E}\right)_{\alpha_{k} \beta_{k}}\right|^{2}\right)^{1 / 2} \cdot\left(\sum_{A_{k}, B_{k}}\left|U\left(g_{b_{k}}\right)_{A_{k} B_{k}}\right|^{2}\right)^{1 / 2}\left(4^{K} \cdot 4^{K} \cdot N^{K} \cdot N^{K}\right)^{1 / 2} e^{l+K+\frac{2 N}{m} K} \\
& \leqq(4 N)^{3 / 2 K} e^{l+K+\frac{2 N}{m} K}=e^{l+0(1) K} .
\end{aligned}
$$


Now

$$
\left|\mathscr{G} \prod_{p \in Q_{0}} \chi\left(g_{p}\right) e^{-A_{\Lambda Q_{Q_{0}}}^{\mathrm{YM}}}\right| \leqq\|\mathscr{G}\|_{\infty} D^{L} e^{\frac{1}{2 g^{2}} D L} \leqq\|\mathscr{G}\|_{\infty} e^{0(1) L} .
$$

From (17), (21), and (22) we get (18).

Lemma 3. For given $K$ and $L$ there are at most $2^{\mid \text {supp } \mathscr{F} \mid} e^{0(1)(K+L)}$ choices of different $\left(\Gamma_{0}, Q_{0}\right)$.

Proof of Lemma. The number $\bar{N}$ in question is bounded by

Number $N_{1}$ of possible ways to draw the bond-graph $\overline{\Gamma_{0} \cup Q_{0}}$ times;

Number $N_{2}$ of possible choices of $\Gamma_{0}$ within a bond-graph times;

Number $N_{3}$ of possible choices of $Q_{0}$ within a bond-graph.

$N_{1} \leqq$ (number of choices of length of the bond-graph) (number of choices of length of connected components of the bond-graph given its total length) -(number of choices of bond-graphs with given length of components) $\leqq(4 L+1)$ $\cdot 2^{\mid \text {supp } \mathscr{F} \mid+K+4 L} \cdot(2 d)^{2(K+L)}$ where $d=4$ is the dimension of the lattice (compare [6, Proof of Proposition 5.1]).

$$
N_{2} \leqq 2^{K}\left(\begin{array}{c}
K+4 L \\
K
\end{array}\right) \leqq 2^{2 K+4 L}
$$

( $2^{K}$ is the number of choices of possible orientations of the bonds of $\Gamma_{0}$ ).

$$
N_{3} \leqq 2^{L}\left(\begin{array}{c}
2(d-1)(K+4 L) \\
L
\end{array}\right) \leqq 2^{L+2(d-1)(K+4 L)} .
$$

Thus

$$
\bar{N} \leqq N_{1} \cdot N_{2} \cdot N_{3} \leqq 2^{|\operatorname{supp} \mathscr{F}|+O(1) \cdot(K+L)} .
$$

Lemma 4. If $m>0(1), g^{2}>0(1)$ and $0(1)$ is big enough then for $X \subset A$

$$
\left|\frac{Z_{A \backslash X}}{Z_{A}}\right| \leqq 2^{|X|}
$$

Proof of Lemma. We proceed as in [10, Proof of Lemma 3.2]. Thus we must show that for $|\Lambda|=N$

$$
\left|\frac{Z_{\Lambda}}{Z_{\Lambda \backslash\left\{x_{0}\right\}}}-1\right| \leqq \frac{1}{2}
$$

provided that (23) holds for $|\Lambda|<N$.

$$
\begin{aligned}
& \left|\frac{Z_{A}}{Z_{\Lambda \backslash\left\{x_{0}\right\}}}-1\right| \leqq \sum_{\substack{\left(\Gamma_{0}, Q_{0}\right) \supset x_{0} \\
\leqq}}\left|R\left(1, \Gamma_{0}, Q_{0}\right)\right| \frac{Z_{\Lambda \backslash} \overline{\Gamma_{0} \cup Q_{0}}}{Z_{A \backslash\left\{x_{0}\right\}}} \\
& \leqq \sum_{\substack{K, L \geqq 0 \\
K+L>0}} e^{O(1)(K+L)}\left(\frac{1}{m}\right)^{K}\left(\frac{1}{g^{2}}\right)^{L} e^{O(1)(K+L)} 2^{K+3 L} \\
& =\frac{1}{1-\frac{2 e^{0(1)}}{m}} \cdot \frac{1}{1-\frac{8 e^{0(1)}}{g^{2}}}-1<\frac{1}{2}
\end{aligned}
$$

if $m$ and $g^{2}$ are big enough. 
The cluster expansion (16) together with the estimates of Lemmas 2 4 (or their versions for the theory with doubled degrees of freedom) give in a standard way (see [6])

Theorem 1. Let $m, g^{2}>0(1)$, with $0(1)$ big enough. Then the cluster expansion for $\langle\mathscr{F}\rangle_{\Lambda}$ converges uniformly in $A$. There exists the thermodynamical limit

$$
\langle\mathscr{F}\rangle=\lim _{\Lambda \rightarrow \mathbb{R}^{d}}\langle\mathscr{F}\rangle_{A}
$$

and the infinite volume theory clusters exponentially.

\section{Confinement of Fermions}

In this section we shall assume that $U$ is an irreducible representation of $G[=U(1)$ or SU(n)] of non-zero $n$-ality, see [10], and that $\chi$ is the trace of $U$. The cluster expansion developed in Section 2 will be used to cast some more light on the problem of confinement of charges connected with gauge invariance. Roughly speaking confinement occurs if the states with charges concentrated in distant regions have very high energy. For some states this will be proven to happen.

Our physical Hilbert space is defined by the usual Osterwalder-Schrader's construction, see [11]. If $\mathscr{F} \in \mathfrak{U}_{\Lambda}^{\mathrm{inv}}$ for some finite subset $\Lambda$ in the positive time halflattice $\mathbb{L}^{+} \subset \mathbb{L}$ and $\Theta \mathscr{\mathscr { F }} \in \mathfrak{U}_{\mathscr{Y}}^{\mathrm{inv}}$ is its time reflection as defined in $[10]$ then (see $[10$, Section II.3])

$$
\langle\Theta \mathscr{F} \cdot \mathscr{F}\rangle \geqq 0 \text {. }
$$

The physical Hilbert space $\mathscr{H}$ is obtained by taking $\mathfrak{U}_{+}^{\text {inv }}:=\underset{\Lambda \subset \mathbb{L}^{+}}{\operatorname{ind}} \lim _{\Lambda} \mathfrak{\mathfrak { r }}^{\text {inv }}$ with the scalar product induced by (24), factorizing out the null subspace and completion. Let $W \mathscr{F}$ denote the canonical image of $\mathscr{F}$ in $\mathscr{H}$. As in [11] one defines a (discrete) semigroup $(S(n))_{n=1,2, \ldots}[S(1)$ is the "transfer matrix" $]$ by

$$
S(n) W \mathscr{F}=W U_{2 n^{2}} \mathscr{F},
$$

where $U_{2 n} \mathscr{F}$ denotes the translation of $\mathscr{F}$ in (Euclidean) time by $2 n$, defined in the obvious way. Now $(S(n))$ is a semigroup of selfadjoint operators in $\mathscr{H}$ of norm $\leqq 1$ (compare [11] - we use the cluster expansion to bound $\left\langle\Theta \mathscr{F} \cdot U_{m} \mathscr{F}\right\rangle$ when $m \rightarrow \infty)$. Also $S(n) \geqq 0$. If $S(1)>0$ then we can define the Hamilton operator

$$
H:=-\frac{1}{2} \log S(1) \text {. }
$$

If $S(1)$ has zero eigenvalue then $H$ is not well defined, however we may speak all the time about expectation values of the energy $\frac{1}{\|X\|^{2}}(X \mid H X)$, for $0 \neq X \in \mathscr{H}$, as given by $\frac{1}{\|X\|^{2}} \int\left(-\frac{1}{2} \log \lambda\right)\left(d E_{S(1)}(\lambda) X \mid X\right)$.

Let for $r=1,3,5, \ldots$

$$
\mathscr{F}_{r}\left(\left(g_{b}\right)\right):=\sum_{\beta, A, B} \psi_{\alpha A}^{2}\left(x_{1}\right)\left(\gamma_{j}^{E}\right)_{\alpha \beta}\left(\prod_{b \subset r_{x_{1} y_{1}}} U\left(g_{b}\right)\right)_{A B} \psi_{\beta B}^{1}\left(y_{1}\right),
$$


where $r_{x_{1} y_{1}}$ is the line segment from $x_{1}$ to $y_{1}$,

$$
x_{1}=\left(\frac{1}{2}, \ldots, \frac{1}{2}\right), \quad y_{1}=\left(\frac{1}{2}, \ldots, \frac{1}{2},-r+\frac{1}{2}, \frac{1}{2}, \ldots, \frac{1}{2}\right), \quad j=1,2,3 \text {. }
$$

Define

$$
X_{r}:=W \mathscr{F}_{r} .
$$

Later we shall show that $X_{r} \neq 0$ in some region of the $m g$ plane. Now by the Jensen inequality

$$
\exp \left[-\frac{2}{\left\|X_{r}\right\|^{2}}\left(X_{r} \mid H X_{r}\right)\right] \leqq \frac{1}{\left\|X_{r}\right\|^{2}}\left(X_{r} \mid e^{-2 H} X_{r}\right),
$$

where, with some abuse of notation, we write $e^{-2 H}$ instead of $S(1)$. Hence to bound the energy of $X_{r}$ from below it is sufficient to find a suitable upper bound on $\frac{1}{\left\|X_{r}\right\|^{2}}\left(X_{r} \mid e^{-2 H} X_{r}\right)$. Introduce

$$
\alpha:=\log m / \log g^{2} .
$$

We shall assume, that $\alpha>3$.

$$
\begin{aligned}
& \left(X_{r} \mid e^{-2 H_{1}} X_{r}\right)=\left\langle\Theta\left(U_{2} \mathscr{F}_{r}\right) \cdot \mathscr{F}_{r}\right\rangle \equiv\left\langle I_{r}\right\rangle \\
& \left\langle I_{r}\right\rangle=\lim _{\Lambda \rightarrow \infty} \sum_{\left(\Gamma_{0}, Q_{0}\right)} R\left(I_{r}, \Gamma_{0}, Q_{0}\right) \frac{Z_{\left.\Lambda \backslash \overline{\Gamma_{0} \cup Q_{0}} \cup \operatorname{supp} I_{r}\right)}}{Z_{\Lambda}},
\end{aligned}
$$

where $R\left(I_{r}, \Gamma_{0}, Q_{0}\right)$ is given by (17). Developing determinants in (17) into powers of $\frac{1}{m}$ we obtain an expansion for $R\left(I_{r}, \Gamma_{0}, Q_{0}\right)$ in terms of fermion paths joining points $\left\{x_{i_{1}}\right\}$ to $\left\{y_{i_{2}}\right\}$ built up of bonds of $\Gamma_{0}$ (with closed loops contribution included), compare [15]. Each bond $b$ in the path contributes a matrix element of $U\left(g_{b}\right)$ as a factor. Subsequent development of $\exp \left(-A_{A \tau_{Q_{0}}}^{\mathrm{YM}}\right)$ into powers of $\frac{1}{g^{2}}$ produces more factors of this type corresponding this time to boundary bonds of plaquettes in $Q_{0}$. Summarizing, $R\left(I_{r}, \Gamma_{0}, Q_{0}\right)$ is a linear combination of $d g_{A}$-integrals of products of matrix elements of $U\left(g_{b}\right)$ for $b$ from $\Gamma_{0}$ or from plaquettes of $Q_{0}$. Now build a one(lattice-)cycle

$$
c_{1}=\sum_{b \subset r_{x_{1} y_{1}}} b+\sum_{b \subset r_{x_{2} y_{2}}} b+\sum_{b \in \Gamma_{0}} b+\sum_{p \in Q_{0}} \sum_{b \subset p} b
$$

where $r_{x_{2} y_{2}}$ is the line segment obtained from $r_{x_{1} y_{1}}$ by translation by -3 in time with change of orientation, see Figure 1. Suppose that one can add to $c_{1}$ a onecycle

$$
d_{1}=\sum_{b \in \Gamma_{0}} n_{b} b+\sum_{p \in Q_{0}} m_{p} \sum_{b \subset p} b \quad\left(n_{b}, m_{p}=0,1, \ldots\right)
$$

such that 


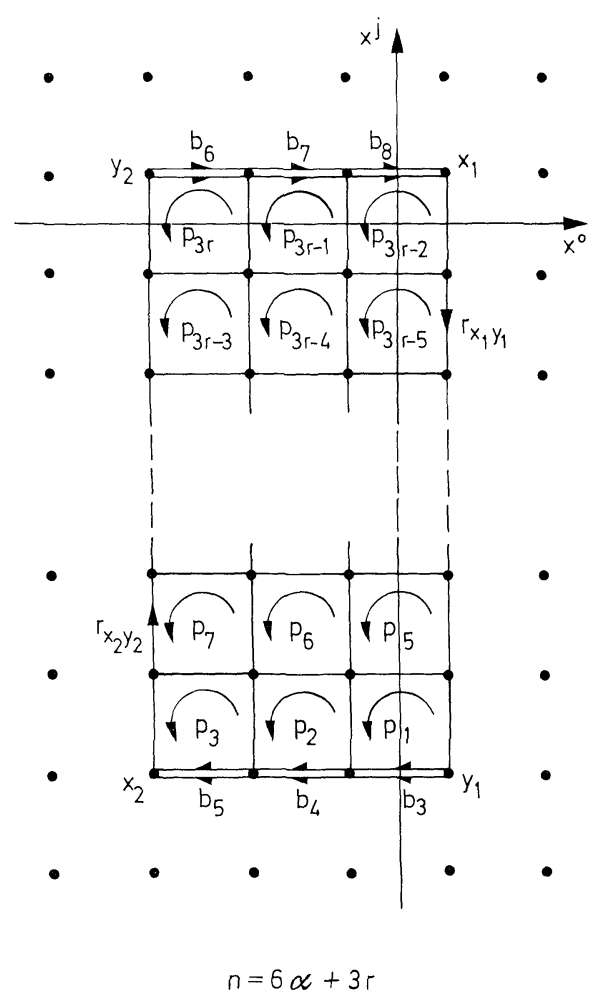

Fig. 1

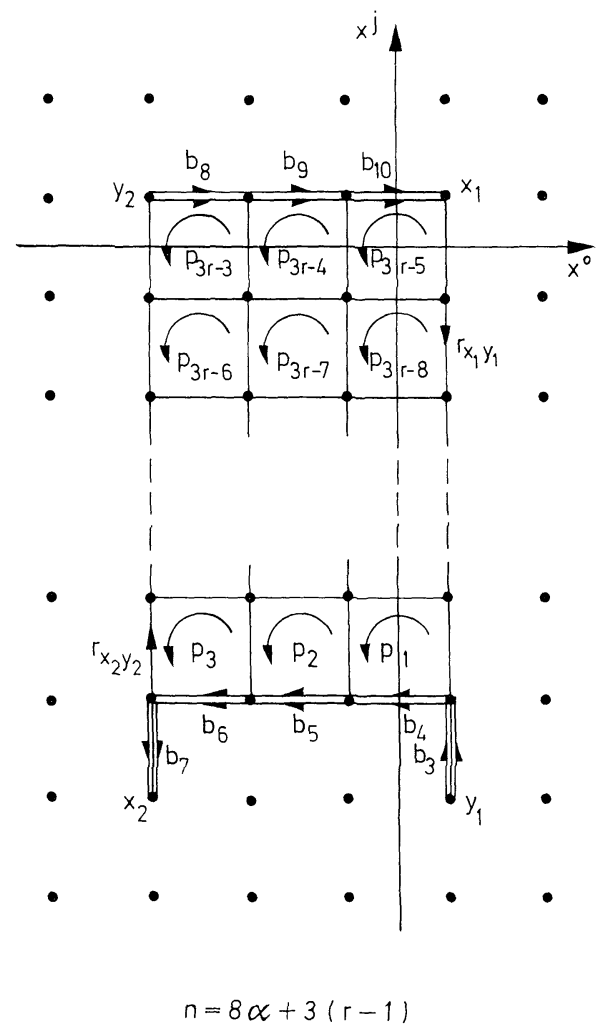

Fig. 2

1. $\partial\left(c_{1}+d_{1}\right)=0$.

2. for each bond $b \in B_{A}^{+}$the overall coefficient at $b$ of $c_{1}+d_{1}$ multiplied by the $n$-ality of $U$ is integer.

It is easily infered from the Peter-Weyl theory that $R\left(I_{r}, \Gamma_{0}, Q_{0}\right)$ can be different from zero only if this holds. It is a very useful observation as it eliminates many terms from the right hand side of (32).

For the time being we shall keep $\alpha$ of (30) fixed, changing $m$ and $g^{2}$. As each term in $R\left(\mathscr{F}, \Gamma_{0}, Q_{0}\right)$ has a factor $\left(\frac{1}{m}\right)^{l+K}\left(\frac{1}{g^{2}}\right)^{L}$ in front, there will be an overall power $\left(\frac{1}{g^{2}}\right)^{(2+K) \alpha+L}$ in front of $R\left(I_{r}, \Gamma_{0}, Q_{0}\right)$. Let

$$
n=n(K, L):=K \alpha+L \text {. }
$$

Suppose that $r \geqq 7$. It is easy to check that all terms of $R\left(I_{r}, \Gamma_{0}, Q_{0}\right)$ with $n<6 \alpha+3 r$ vanish. The lowest non-vanishing term with $n=n_{0} \equiv 6 \alpha+3 r$ corresponds to $\left(\Gamma_{0}, Q_{0}\right)$ as on Figure 1. Next terms have $n=8 \alpha+3(r-1)$ (one of them is pictured 
on Fig. 2). Thus

$$
\left(X_{r} \mid e^{-2 H} X_{r}\right) \leqq \sup _{\Lambda} \sum_{\left(\Gamma_{0}, Q_{0}\right)}\left|R\left(I_{r}, \Gamma_{0}, Q_{0}\right)\right| \frac{Z_{\Lambda \backslash\left(\overline{\Gamma_{0} \cup Q_{0}} \cup \operatorname{supp} I_{r}\right)}}{Z_{\Lambda}} \mid,
$$

where the sum is taken over all $\left(\Gamma_{0}, Q_{0}\right)$ such that $n(K, L) \geqq 6 \alpha+3 r$.

(34) produces an upper bound upon $\left(X_{r} \mid e^{-2 H} X_{r}\right)$.

\section{Proposition 1.}

$$
\left(X_{r} \mid e^{-2 H} X_{r}\right) \leqq\left(\frac{0(1)}{g^{2}}\right)^{8 \alpha+3 r}
$$

provided $r \geqq 7, \frac{0(1)}{g^{2}}<1$ and $0(1)$ is big enough.

Proof. From (34) and Lemmas 2 and 3

$$
\begin{aligned}
\left(X_{r} \mid e^{-2 H} X_{r}\right) \leqq & 4 N^{2} e^{2} 4^{\left|\operatorname{supp} I_{r}\right|} \sum_{\substack{K, L \geq 0 \\
n(K, L) \geqq n_{0}}} \frac{\left(2 e^{O(1)}\right)^{K}\left(8 e^{O(1)}\right)^{L}}{\left(g^{2}\right)^{(2+K) \alpha+L}} \\
\leqq & 4 N^{2} e^{2} 4^{2(r+1)}\left(\frac{1}{g^{2}}\right)^{2 \alpha}\left[\sum_{L=0 K=-\left[\left(L-n_{0}\right) / \alpha\right]}^{\left[n_{0}\right]}\left(\frac{O(1)}{g^{2}}\right)^{\alpha K+L}\right. \\
& \left.+\sum_{L=\left[n_{0}\right]+1}^{\infty} \sum_{K=0}^{\infty}\left(\frac{O(1)}{g^{2}}\right)^{\alpha K+L}\right] \leqq\left(\frac{O(1)}{g^{2}}\right)^{2 \alpha+n_{0}} .
\end{aligned}
$$

Here $[x]$ denotes the biggest integer less or equal $x$ and (36) holds provided, say, $\frac{0(1)}{g^{2}}<1$ and $0(1)$ is large.

We shall also need a lower bound on $\left\|X_{r}\right\|$. The point is that it is sufficient to bound from below $\left\|X_{5}\right\|$ for example and then use the following

Proposition 2. For $r \geqq 7$

$$
\frac{1}{N^{r-5}}\left\|X_{5}\right\| r-3 \leqq\left\|X_{3}\right\|^{r-5}\left\|X_{r}\right\|^{2} .
$$

Proof. We use a sort of Nelson symmetry argument [14]. Following the construction of $[10]$ introduce an axial gauge in which $g_{b}=1$ for $b$ in the direction of the $j$-th axis. Thus consider the algebras $\mathfrak{U}_{A}^{\text {ax }}$ of continuous mappings on $\underset{\substack{b \in B_{A}^{+} \\ b \\ X_{j-\text { his }}}}{ } G$ with values in the Grassmann algebra $\mathscr{A}_{\Lambda}$. For $J \in \mathfrak{Q}_{\Lambda}^{a x}$ define the expectation $\langle J\rangle_{A}^{\mathrm{ax}}$ for which the cluster expansion holds (all estimates of Section 2 hold mutatis mutandis). So there exists an exponentially clustering infinite volume state \langle\rangle$^{\mathrm{ax}}=\lim _{\Lambda \rightarrow \mathbb{R}^{d}}\langle\rangle_{\Lambda}^{\mathrm{ax}}$ defined on indlim $\mathfrak{U}_{\Lambda}^{\mathrm{ax}}$. Now introduce an operator $\Theta_{j}$ of reflection with respect to the plane $x^{j}=0$, similarly as $\Theta$ was introduced in [10] (one uses $\gamma_{j}^{E}$ instead of $\gamma_{0}^{E}$ in the following way: $\Theta_{j} \psi_{\alpha A}^{1}(x)$ 
$=\sum_{\beta} \psi_{\beta A}^{2}\left(\vartheta_{j} x\right)\left(\gamma_{j}^{E}\right)_{\beta \alpha}, \Theta_{j} \psi_{\alpha A}^{2}(x)=\sum_{\beta}\left(\gamma_{j}^{E}\right)_{\alpha \beta} \psi_{\beta A}^{1}\left(\vartheta_{j} x\right)$, where $\vartheta_{j} x$ is the reflected lattice site). Mimicking the proof of [10, Theorem 2.1] we conclude that whenever $J \in \mathfrak{P}_{\Lambda}^{\text {ax }}$ with $\Lambda$ composed of sites with positive $j$-th coordinate then

$$
\left\langle\Theta_{j} J \cdot J\right\rangle^{\mathrm{ax}} \geqq 0 \text {. }
$$

As before, we produce a Hilbert space $\mathscr{H}_{j}^{\text {ax }}$ using $\left\langle\Theta_{j} J \cdot J\right\rangle^{\text {ax }}$ as a scalar product in $\mathfrak{U}_{j+}^{\mathrm{ax}}:=\operatorname{ind} \lim \mathfrak{U}_{\Lambda}^{\mathrm{ax}}$, where we take only $\Lambda$-s with positive $j$-th coordinate. If $W_{j}^{\mathrm{ax}} J$ denotes the canonical image of $J$ in $\mathscr{H}_{j}^{\text {ax }}$ and $U_{2 n}^{j}$ the translation by $2 n$ in the $j$-th direction acting on $\mathfrak{U}_{j+}^{\mathrm{ax}}$ then again

$$
\left\langle\Theta_{j} J \cdot U_{2 n}^{j} J\right\rangle^{\mathrm{ax}}=\left(W_{j}^{\mathrm{ax}} J \mid S_{j}^{\mathrm{ax}}(n) W_{j}^{\mathrm{ax}} J\right),
$$

where $\left(S_{j}^{\text {ax }}(n)\right)_{n=1,2, \ldots}$ is a selfajoint semigroup of non-negative operators in $\mathscr{H}_{j}^{\text {ax }}$. Now the Hölder inequality for the spectral measure of $S_{j}^{\mathbf{a x}}(1)$ gives

$\left(\left\langle\Theta_{j} J \cdot U_{4} J\right\rangle^{\mathrm{ax}}\right)^{(r-3) / 2} \leqq\left(\left\langle\Theta_{j} J \cdot U_{2} J\right\rangle^{\mathrm{ax}}\right)^{(r-5) / 2}\left\langle\Theta_{j} J \cdot U_{r-1} J\right\rangle^{\mathrm{ax}}$

for odd $r \geqq 7$.

For $\mathscr{F}_{r}$ given by (27)

$$
\begin{aligned}
\left\langle\Theta \mathscr{F}_{r} \cdot \mathscr{F}_{r}\right\rangle= & \sum_{A, B}\left\langle\sum_{\beta, \gamma, \delta} \psi_{\gamma B}^{2}\left(\vartheta y_{1}\right)\left(\gamma_{j}^{E}\right)_{\gamma \alpha}\left(\gamma_{0}^{E}\right)_{\alpha \delta} \psi_{\delta B}^{1}\left(\vartheta x_{1}\right)\right. \\
& \cdot \psi_{\alpha A}^{2}\left(\left(x_{1}\right)\left(\gamma_{j}^{E}\right)_{\alpha \beta} \psi_{\beta A}^{1}\left(y_{1}\right)\right\rangle^{\mathrm{ax}} \\
= & \sum_{A, B}\left\langle\Theta_{j} J_{A B} \cdot U_{r-1} J_{A B}\right\rangle^{\mathrm{ax}},
\end{aligned}
$$

where

$$
J_{A B}:=\sum_{\beta} \psi_{\alpha A}^{2}\left(x_{1}\right)\left(\gamma_{0}^{E}\right)_{\alpha \beta} \psi_{\beta B}^{1}\left(\vartheta x_{1}\right) .
$$

Combining (39) and (38) we obtain

$$
\frac{1}{N^{r-5}}\left\langle\Theta \mathscr{F}_{5} \cdot \mathscr{F}_{5}\right\rangle^{(r-3) / 2} \leqq\left\langle\Theta \mathscr{F}_{3} \cdot \mathscr{F}_{3}\right\rangle^{(r-5) / 2}\left\langle\Theta \mathscr{F}_{r} \cdot \mathscr{F}_{r}\right\rangle
$$

which is (37).

Proposition 3. $\left\|X_{5}\right\|^{2} \geqq \frac{1}{O(1)}\left(\frac{1}{g^{2}}\right)^{4 \alpha+5}$ if $\frac{O(1)^{\alpha}}{g^{2}}<1$ and $O(1)$ is big enough.

Proof. Let us notice that in the cluster expansion for $\left\langle\Theta \mathscr{F}_{5} \cdot \mathscr{F}_{5}\right\rangle_{A}$ all terms with $n$ $<2 \alpha+5$ vanish. There is a non-vanishing term for $n=2 \alpha+5$ (see Fig. 3) and other terms have $n \geqq n_{1} \equiv 4 \alpha+4$. Now

$$
\begin{aligned}
& \sup _{\Lambda}\left|\sum_{\substack{\left(\Gamma_{0}, Q_{0}\right) \\
n(K, L) \geqq n_{1}}} R\left(\Theta \mathscr{F}_{5} \cdot \mathscr{F}_{5}, \Gamma_{0}, Q_{0}\right) \frac{Z_{\Lambda \backslash\left(\overline{\Gamma_{0} \cup Q_{0}} \cup \operatorname{supp} \Theta \mathscr{F}_{5} \cdot \mathscr{F}_{5}\right)}}{Z_{A}}\right| \\
& \leqq\left(\frac{O(1)}{g^{2}}\right)^{6 \alpha+4} \quad \text { if } \quad \frac{0(1)}{g^{2}}<1
\end{aligned}
$$


and $0(1)$ is big, which is proven the same way (35) was. Thus

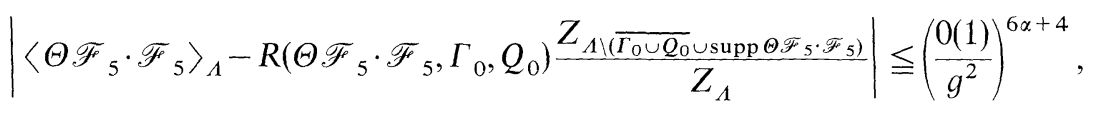

where $\left(\Gamma_{0}, Q_{0}\right)$ is chosen according to Figure 3 (and $\Lambda$ is big enough).

Define

$$
R_{0}\left(\mathscr{F}, \Gamma_{0}, Q_{0}\right):=\frac{1}{m^{l+K} g^{2 L}} \lim _{\substack{m \rightarrow \infty \\ g^{2} \rightarrow \infty}} m^{l+K} g^{2 L} R\left(\mathscr{F}, \Gamma_{0}, Q_{0}\right)
$$

$$
\begin{aligned}
& \cdot \sum_{\substack{\alpha_{i}, \beta_{k}, A_{1}, B_{k} \\
i=1,2,3,4}}\left\lfloor\left(\prod_{k=3}\left(\gamma_{b_{k}}^{\mu}\right)_{\alpha_{k} \beta_{k}} U\left(g_{b_{k}}\right)_{A_{k} B_{k}}\right\rfloor\right. \\
& \cdot \operatorname{det}_{i_{1} i_{2}}^{4 \times 4}\left[\delta_{\beta_{i_{1}}, \alpha_{i_{2}}} \delta_{B_{\imath_{1}}, A i_{2}} \delta_{y_{i_{1}}, x i_{2}}\right]\left(\gamma_{j}^{E}\right)_{\alpha_{1} \beta_{1}}\left(\gamma_{0}^{E} \gamma_{j}^{E}\right)_{\alpha_{2} \alpha_{1}}\left(\gamma_{0}^{E}\right)_{\alpha_{1} \beta_{2}} \delta_{\alpha_{1}, \alpha} \\
& \left.\cdot\left(\prod_{b \subset r_{x_{1} y_{1}}} U\left(g_{b}\right)\right)_{A_{1} B_{1}}\left(\prod_{b \subset r_{x_{2} y_{2}}} U\left(g_{b}\right)\right)_{A_{2} B_{2}}\right] \prod_{m=1}^{5} \chi\left(g_{p_{m}}\right) \\
& =\frac{1}{2^{7}\left(g^{2}\right)^{4 \alpha+5}} \int d g_{A} \operatorname{Tr}\left(\left(\prod_{b \subset r_{x_{1} y_{1}}} U\left(g_{b}\right)\right) U\left(g_{b_{3}}\right)\left(\prod_{b \subset \boldsymbol{r}_{x_{2} y_{2}}} U\left(g_{b}\right)\right) U\left(g_{b_{4}}\right)\right) \prod_{m=1}^{5} \chi\left(g_{p_{m}}\right) \\
& =\frac{1}{2^{7} N^{4}}\left(\frac{1}{g^{2}}\right)^{4 \alpha+5} \text {. }
\end{aligned}
$$

The last line was obtained by subsequent use of orthogonality relations between the matrix elements of $U$.

\section{Lemma 5.}

$$
\begin{aligned}
& \left|R\left(\Theta \mathscr{F}_{5} \cdot \mathscr{F}_{5}, \Gamma_{0}, Q_{0}\right) \frac{Z_{\Lambda \backslash\left(\overline{\Gamma_{0} \cup Q_{0}} \cup \operatorname{supp} \Theta \mathscr{F}_{5} \cdot \mathscr{F}_{5}\right)}}{Z_{\Lambda}}-R_{0}\left(\Theta \mathscr{F}_{5} \cdot \mathscr{F}_{5}, \Gamma_{0}, Q_{0}\right)\right| . \\
& \quad \leqq\left(\frac{0(1)}{g^{2}}\right)^{4 \alpha+6}
\end{aligned}
$$

for $\frac{0(1)}{g^{2}}<1$ and $0(1)$ big enough.

Proof of Lemma. First we need a more refined version of Lemma 4. For $X \subset A$ we have

$$
1-\frac{Z_{\Lambda \backslash X}}{Z_{\Lambda}}=\sum_{\left(\Gamma_{0}, Q_{0}\right)} R\left(1, \Gamma_{0}, Q_{0}\right) \frac{Z_{\left.\Lambda \backslash \overline{\Gamma_{0} \cup Q_{0}} \cup X\right)}}{Z_{\Lambda}},
$$




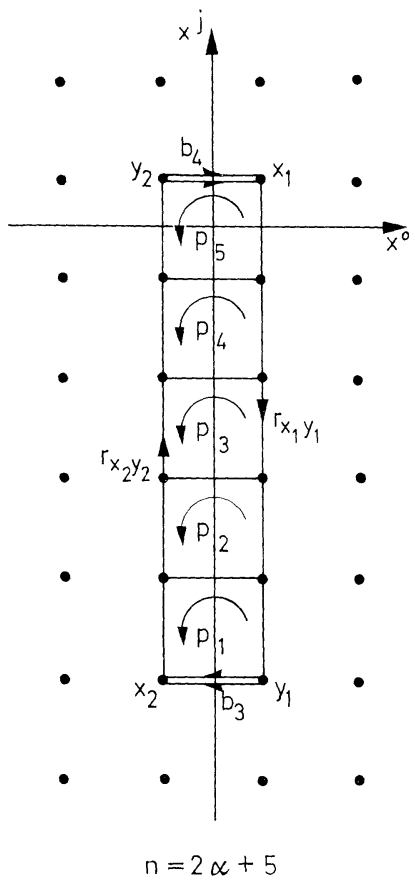

Fig. 3

where we sum over $\left(\Gamma_{0}, Q_{0}\right)$ such that $\overline{\Gamma_{0} \cup Q_{0}}$ has no connected component disjoint with $X$. Using estimates of Lemmas 2-4 we get

$$
\left|1-\frac{Z_{\Lambda \backslash X}}{Z_{\Lambda}}\right| \leqq \sum_{\substack{K, L \geqq 0 \\ K+L>0}} 4^{|X|}\left(\frac{2 e^{0(1)}}{g^{2}}\right)^{K}\left(\frac{8 e^{0(1)}}{g^{2}}\right)^{L} \leqq 4^{|X|} \frac{0(1)}{g^{2}}
$$

for $0(1)$ on the right hand side big enough and $\frac{0(1)}{g^{2}}<1$. Now

$$
\begin{aligned}
& \operatorname{det}_{i_{1} i_{2}}^{(l+K) \times(l+K)}\left[\delta_{\beta_{i_{1}}, \alpha_{i_{2}}} \delta_{B_{i_{1}}, A_{i_{2}}} \delta_{y_{i_{1}}, x_{l_{2}}}\right] \\
& \quad-\operatorname{det}_{i_{1} i_{2}}^{(l+K) \times(l+K)}\left[\left(1-\frac{1}{m} K_{\Lambda s_{\Gamma_{0}}}\right)^{-1} \beta_{i_{1} B_{i_{1}} y_{i_{1}}, \alpha_{i_{2}} A_{i_{2}} x_{i_{2}}}\right] \operatorname{det}\left(1-\frac{1}{m} K_{\Lambda s_{\Gamma_{0}}}\right) \mid \leqq \frac{O(1)}{g^{2}}(46)
\end{aligned}
$$

if $\frac{0(1)}{g^{2}}<1,0(1)$ big, by (19), (20) and the Cauchy integral formula. Also

$$
\left|1-e^{-A_{\Lambda \tau}^{Y M} Q_{0}}\right| \leqq \frac{0(1)}{g^{2}}
$$

if $\frac{O(1)}{g^{2}}<1$ and $O(1)$ is big. 


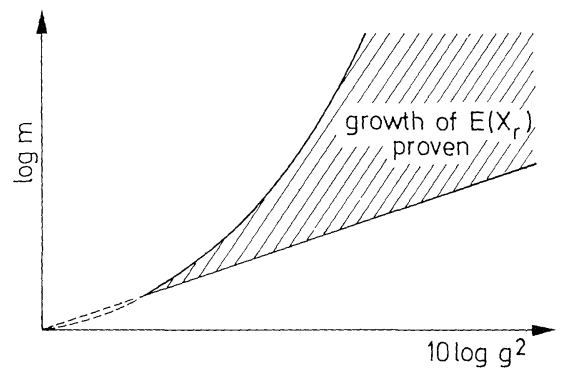

Fig. 4

Estimating the left hand side of (44) step-wise using the triangle inequality and (45)-(47) we obtain (44).

Now (41) and Lemma 5 show that

$$
\left|\left\langle\Theta \mathscr{F}_{5} \cdot \mathscr{F}_{5}\right\rangle-R_{0}\left(\Theta \mathscr{F}_{5} \cdot \mathscr{F}_{5}, \Gamma_{0}, Q_{0}\right)\right| \leqq\left(\frac{0(1)}{g^{2}}\right)^{4 \alpha+6}
$$

again for $\frac{O(1)}{g^{2}}<1$ and $0(1)$ big enough. (43) and (48) yield Proposition 3.

From Propositions 2 and 3 we conclude that $X_{r} \neq 0$ for $g^{2}$ sufficiently large.

We shall need one more estimate which is proven in the same way as Proposition 1.

Lemma 6. $\left\|X_{3}\right\|^{2} \leqq\left(\frac{0(1)}{g^{2}}\right)^{4 \alpha+3}$ for $\frac{O(1)}{g^{2}}<1,0(1)$ big.

From Propositions 1-3 and Lemma 6 we obtain

$$
\begin{aligned}
& \frac{1}{\left\|X_{r}\right\|^{2}}\left(X_{r} \mid e^{-2 H} X_{r}\right) \leqq N^{r-5}\left\|X_{5}\right\|^{3-r}\left\|X_{3}\right\|^{r-5}\left(X_{r} \mid e^{-2 H} X_{r}\right) \\
& \leqq N^{r-5} 0(1)^{(r-3) / 2}\left(\frac{1}{g^{2}}\right)^{(4 \alpha+5)(3-r) / 2}\left(\frac{0(1)}{g^{2}}\right)^{(4 \alpha+3)(r-5) / 2}\left(\frac{0(1)}{g^{2}}\right)^{8 \alpha+3 r} \\
& \leqq\left(\frac{0(1)^{\alpha}}{g^{2}}\right)^{2 r}
\end{aligned}
$$

Thus for $\frac{0(1)^{\alpha}}{g^{2}}<1,0(1)$ big enough,

$$
E\left(X_{r}\right) \equiv \frac{1}{\left\|X_{r}\right\|^{2}}\left(X_{r} \mid H X_{r}\right) \geqq r \log \left(g^{2} / 0(1)^{\alpha}\right) .
$$

Hence $E\left(X_{r}\right)$ grows if $g^{2}>0(1)^{\alpha}, \alpha>3$, i.e. for (see Fig. 4) $1<\left(g^{2}\right)^{3}<m<\left(g^{2}\right)^{\varepsilon \log g^{2}}$ and $\varepsilon=\varepsilon(N)$ small enough.

We have proven

Theorem 2. There exists $0<\varepsilon=\varepsilon(N)$ such that for $1<\left(g^{2}\right)^{3}<m<\left(g^{2}\right)^{\varepsilon \log g^{2}}$

$$
E\left(X_{r}\right) \geqq\left(\log g^{2}-\frac{1}{\varepsilon} \frac{\log m}{\log g^{2}}\right) r .
$$


Acknowledgements. I am grateful to Professor W. Zimmermann for hospitality at the Max-PlanckInstitut für Physik und Astrophysik in Munich. Discussions with Professors H. Reeh and S. Schlieder were of great help to me.

\section{References}

1. Balian, R., Drouffe,J.M., Itzykson, C.: Gauge fields on a lattice. I-III. Phys. Rev. D 10, 3376--3395 (1974), 2098--2103, and 2104-2119 (1975)

2. Callan, C.G., Dashen, R.F., Gross, D.J.: The structure of the gauge theory vacuum. Phys. Lett. 63B, 334-340 (1976)

3. Callan, C.G., Dashen, R.F., Gross,D.J.: A mechanism for quark confinement. Phys. Lett. 66B, 375-381 (1977)

4. Cooper,A., Rosen,L.: The weakly coupled Yukawa ${ }_{2}$ field theory: cluster expansion and Wightman axioms. Preprint (1976)

5. Glimm,J., Jaffe, A.: Instantons in a $U(1)$ lattice gauge theory: a Coulomb dipole gas. Preprint (1977)

6. Glimm,J., Jaffe, A., Spencer, T.: The particle structure of the weakly coupled $P(\varphi)_{2}$ model and other applications of high temperature expansions. I, II. In : Constructive quantum field theory. Lecture notes in physics, Vol. 25, pp. 132-198 and 199-242. Berlin-Heidelberg-New York: Springer 1973

7. Kogut,J., Susskind,L.: Hamiltonian formulation of Wilson's lattice gauge theories. Phys. Rev. D11, 395--408 (1975)

8. Magnen,J., Sénéor, R.: The Wightman axioms for the weakly coupled Yukawa model in two dimensions. Commun. math. Phys. 51, 297--313 (1976)

9. Osterwalder, K.: Yang-Mills fields on the lattice. Preprint (1976)

10. Osterwalder,K., Seiler,E.: Gauge field theories on the lattice. Preprint (1977)

11. Osterwalder,K., Schrader, R.: Axioms for Euclidean Green's functions. Commun. math. Phys. 31, 83-112 (1973)

12. Polyakov, A. M.: Compact gauge fields and the infrared catastrophe. Phys. Lett. 59 B, $82--87$ (1975)

13. Polyakov, A. M.: Quark confinement and topology of gauge theories. Nucl. Phys. B120, 429 -458 (1977)

14. Simon, B.: The $P(\varphi)_{2}$ Euclidean (quantum) field theory. Princeton New Jersey: Princeton University Press 1974

15. Wilson, K. G.: Confinement of quarks. Phys. Rev. D10, 2445-2459 (1974)

Communicated by A. Jaffe

Received October 27, 1977; in revised form January 26, 1978

\section{Note Added in Proof}

In fact it is not so difficult to extend our result to states $X_{r}^{\prime}$ of the form

$$
X_{r}^{\prime}=W\left(\sum_{A, B} \psi_{\alpha A}^{2}\left(x_{1}\right)\left(\prod_{b C r_{\alpha_{1} \nu_{1}}} U\left(g_{b}\right)\right)_{A B} \psi_{\beta B}^{1}\left(y_{1}\right)\right)
$$

and dismiss our assumption on spins of the fermion and the antifermion. We must only notice that in the cluster expansion for $\left\|X_{r}^{\prime}\right\|^{2}$ all terms for which $\Gamma_{0}$ does not connect $\left\{x_{1}, y_{2}\right\}$ with $\left\{x_{2}, y_{1}\right\}$ coincide with their counterparts in the cluster expansion for $\left\|X_{r}\right\|^{2}$. The sum of the other terms is bounded by $0(1)^{r} /\left(g^{2}\right)^{(2+2 r) \alpha}$ for small $g^{2}$. However our bounds give

$$
\left\|X_{r}\right\|^{2} \geqq \frac{1}{0(1)^{\alpha r}\left(g^{2}\right)^{r+4 \alpha}}
$$

if $0(1)^{\alpha} / g^{2}<1$ and $O(1)$ is big enough. Thus the similar lower bound holds for $\left\|X_{r}^{\prime}\right\|^{2}$ and yields, together with an analog of Proposition 1, a linear lower bound on the energy of $X_{r}^{\prime}$. 
